\title{
The State of the Art of Rare Gas Excimer Lasers and Lamps as a Light Source for Giga-bit Lithography
}

\author{
Wataru Sasaki, Kou Kurosawa, Shoichi Kubodera, Junji Kawanaka \\ Photon Science Center, Faculty of Engineering, University of Miyazaki \\ Nishi 1-1, Gakuen Kibanadai, Miyazaki 889-2192
}

\begin{abstract}
Coherent and incoherent light sources using rare gas excimers are reviewed from a point of view of post ArF lithography. Present status of electron beam pumped rare gas excimer lasers are reported. Potentialities of rare gas excimer lasers by discharge pumping are discussed. We have obtained laser gain of $2.2 \times 10^{-3} \mathrm{~cm}^{-1}$ in $\mathrm{Kr}$ discharges which was very close to the threshold gain. It is pointed out that gas excimer lasers are suitable for light sources for next generation lithography after ArF lithography in the 21th century, when a compact excitation method is established. A jet discharge excimer lamps are also discussed from a point of view of an incoherent light source for lithography.

Keywords: rare gas excimer, vuv radiation, electron beam excitation, discharge excitation, jet discharge, lithography light source
\end{abstract}

\section{Introduction}

It has been long time to introduce excimer lasers in mass production lines of LSI industry. Now $\mathrm{KrF}$ excimer lasers of $248 \mathrm{~nm}$ wavelength are begun to use for lithography of 64 MDRAM and $250 \mathrm{MHz}$ CPU where $0.3 \mu \mathrm{m}$ design rules are required. An ArF laser with $193 \mathrm{~nm}$ wavelength is a strong candidate for $0.25 \mu \mathrm{m}$ optical lithography in the next century.

There have been many discussions for exploring the potentialites of advanced optical lithography below $193 \mathrm{~nm}$ wavelength. Optical lithography industry will jump to soft $x$-ray wavelength to obtain even higher spatial resolution. On the other hand, a compact VUV laser could be a strong alternative for post $\mathrm{ArF}$ laser lithography.
In this letter the state of the art of VUV lasers is reviewed with a focus on rare gas excimer lasers. Rare gas excimers have long been one of the very few laser media in the VUV spectral region.[1] The emission wavelength of $\mathrm{Ar}_{2}{ }^{*}$ is $126 \mathrm{~nm}$ which is long enough to use transmission optical elements such as $\mathrm{MgF}_{2}$ and $\mathrm{LiF}$. $\mathrm{A} \mathrm{Kr}_{2}{ }^{*}$ laser has an even longer emission wavelength centered at $146 \mathrm{~nm}$ which reduces the conditions for optics and would become a competitor to an $\mathrm{F}_{2}$ laser at $157 \mathrm{~nm}$. Rare gases are chemically stable which shows a strong contrast to chemically active fluorine in a $F_{2}$ laser. In addition to the well known homo-nuclear rare gas excimer continuum, emission from hetero-nuclear rare gas excimers were obtained by mixing a trace of heavier rare gas. 


\section{Rare gas excimer lasers}

2-1. Electron beam pumped rare gas excimer lasers

Rare gas excimer lasers are attractive sources for applications not only in optical lithography but also in various industrial fields such as photochemistry and new types of materials processing. Electron beam pumping however, is the only excitation method to realize the laser oscillation of rare gas excimers. We have been developing a high peak power $\mathrm{Ar}_{2}{ }^{*}$ and $\mathrm{Kr}_{2}{ }^{*}$ lasers using electron beam pumping for fusion plasma diagnosis.[2,3] A part of a typical electron beam machine consisting of a Marxgenerator, Blumlein-type pulse-forming circuit and a diode is shown in Fig. 1. The laser gas contained in the tube which was an anode of a coaxial-type diode was pumped by an electron beam with a peak voltage of $700 \mathrm{keV}$ and a pulse duration time of $90 \mathrm{~ns}$ in FWHM. The coaxial diode consists of a cathode with $40 \mathrm{~cm}$ length and a concentric anode made up of a stainless steel

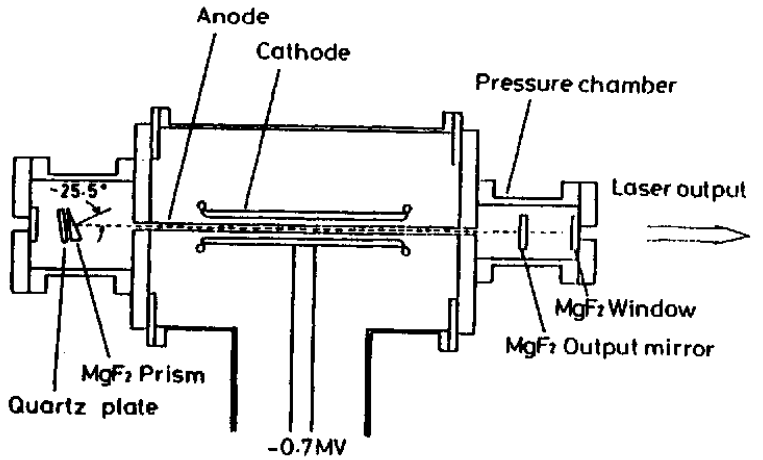

Fig. 1. Schematic diagram of a coaxialdiode and a laser resonator.

tube of $5 \mathrm{~mm}$ outer diameter and $80 \mu \mathrm{m}$ wall thickness (Kobe Steel Co. Ltd.). The ends of anode pipe opened into the high pressure chamber, where the laser mirrors of resonant cavity were placed. The resonant cavity could be composed of an uncoated $\mathrm{MgF}_{2}$ plate as an output mirror and a $\mathrm{Al}, \mathrm{SiC}$ or $\mathrm{Si}$ plate as a back mirror. Lasing conditions of rare gas excimer lasers are summarized in Table 1 . Our efforts have been focused on the $\mathrm{Ar}$ and $\mathrm{Kr}$ excimer

Table 1 Present status of rare gas excimer lasers

\begin{tabular}{ccccc}
\hline Species & Wavelength[nm] & Peak power[MW] & Band width[nm] & Resonator \\
\hline Ar & 126 & 16 & 1 & SiC-MgF2 \\
& $124-128$ & $>1$ & 0.3 & 16 deg. MgF2 prism \\
$\mathrm{Kr}$ & 147 & 7 & 1 & $\mathrm{Si}-\mathrm{MgF} 2$ \\
& 145.5 (tuned) & 3.5 & 0.1 & 32 deg. MgF2 prism \\
$\mathrm{Xe}$ & 172 & 400 & - & - \\
& $170-176^{*}$ & 1 & 0.1 & $\mathrm{MgF} 2$ prism \\
\hline
\end{tabular}

* Ref. [12] 
lasers with high peak power which oscillate in the vicinity of $126 \mathrm{~nm}$ and $146 \mathrm{~nm}$. The maximum peak output power of the Ar excimer laser was $16 \mathrm{MW}$ with $1 \mathrm{~nm}$ spectral width when using a $\mathrm{SiC}$ back mirror and $\mathrm{MgF}_{2}$ output mirror. A Si back mirror and $\mathrm{MgF}_{2}$ output mirror worked best for the $\mathrm{Kr}$ excimer laser, yielding $7 \mathrm{MW}$ in a $1 \mathrm{~nm}$ band width. The largest peak power Xe excimer laser, developed by Ault et al. [4], yielded 400 MW, but details of this laser have not been published.

Broad band gain profiles of the rare gas excimer lasers permit laser wavelength tuning by using dispersive optics. $\mathrm{A} \mathrm{MgF}_{2}$ prism with 16 degrees apex angle was inserted in the cavity of the Ar excimer laser to yield a tuning range of $124 \mathrm{~nm}$ to $128 \mathrm{~nm}$ at a line width of $0.3 \mathrm{~nm}$.[5] A larger apex angle $\mathrm{MgF}_{2}$ prism with 32 degrees was used in the $\mathrm{Kr}$ excimer laser to provide the same dispersion. The tuning range was not fully tested, but a peak power of $3.5 \mathrm{MW}$ in a narrow line width of $0.1 \mathrm{~nm}$ was produced.[6]

Despite their high peak power operation in the VUV spectral region, electron beam excitation method may be unsuitable for certain applications such as optical lithography in the next generation, because they require a rather large facility to be operated, resulting in a low repetition rate and low average power of a laser beam. A compact rare gas excimer laser with high repetition operation is in high demand for an optical lithography. Various excitation methods have thus been studied to demonstrate compact rare gas excimer lasers.[7,8,9] We have attempted to obtain a laser oscillation of rare gas excimers by a discharge excitation.
2-2. Discharge pumped rare gas excimer lasers

A discharge pumped rare gas excimer laser is one of the favorable light sources for an optical lithography in the next generation. We have investigated various types of discharge excitations of rare gas excimer lasers. $[8,9,10]$ In order to establish laser oscillation of rare gas excimers by discharge pumping it is considered that stable arc free discharges should be accomplished in a high pressure rare gas.

Fig. 2 shows a schematic diagram of a discharge tube and a laser cavity used in our experiments. Although the discharge tube seems to be the same as those of rare gas halide excimer lasers, some improvements have been done to

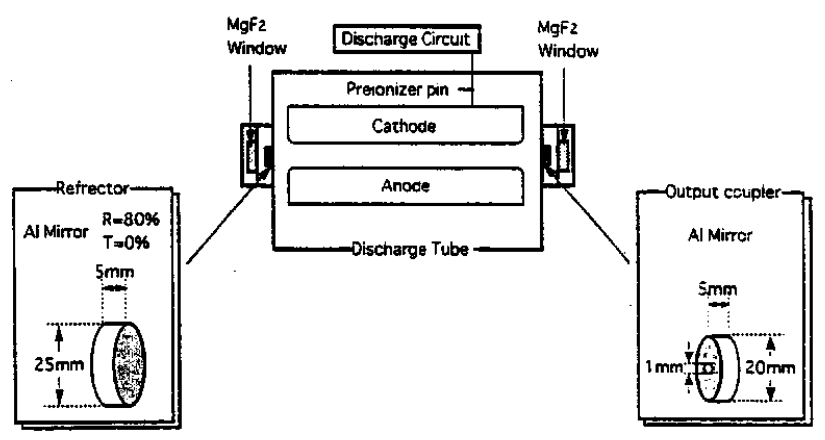

Fig. 2. A schematic diagram of a discharge tube and cavity mirrors.

attain an arc free discharge in a high pressure rare gas. In order to obtain strong photopreionization 102 pairs of pre-discharge pins were set at both sides of main electrodes in the high pressure gas container up to $10 \mathrm{~atm}$. The inductance of the main discharge circuit was reduced as low as $2.6 \mathrm{nH}$ to obtaine first rise time discharges. The electrodes were $108 \mathrm{~cm}$ length 
with $2 \mathrm{~cm}$ width having a Chang-shape structure. The discharge gap was set at $6 \mathrm{~mm}$. A laser cavity was composed of a pair of $\mathrm{Al}$ mirrors and set in the pressure chamber at the both ends of the electrodes. One of the Al mirror had a small hole of $1 \mathrm{~mm}$ diameter at the center for output coupling. The reflectivity of the Al back mirror was $80 \%$ at $147 \mathrm{~nm}$ and the effective reflectivity of the output coupler is estimated about $40 \%$.

Fig. 3 shows a time-integrated emission spectra from a discharge region observed through the coupling hole. A broad emission centered at $147 \mathrm{~nm}$ was identified as an emission from $\mathrm{Kr}_{2}{ }^{*}$.

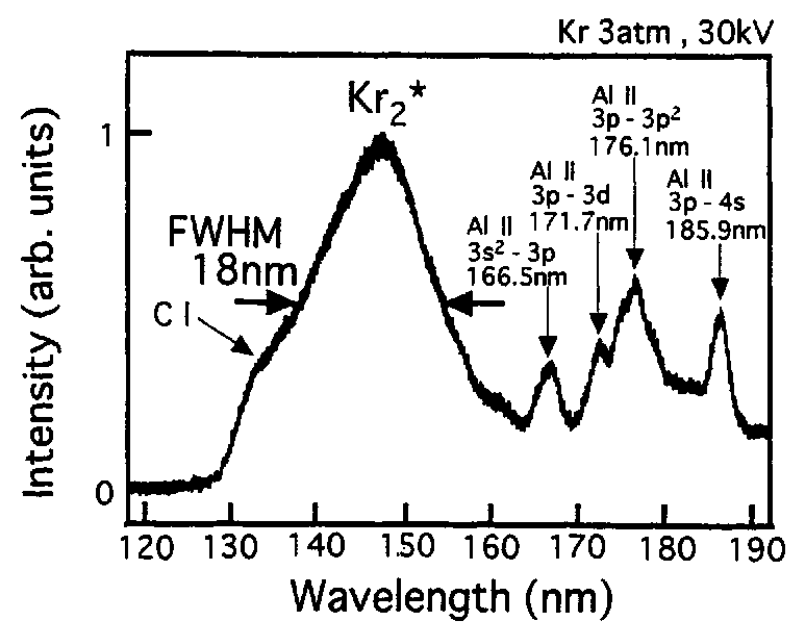

Fig. 3. Time-integrated emission spectra from a krypton discharge.

Several lines in longer wavelength were considered as emissions from $\mathrm{Al}$ ions which were sputtered from the pre-discharge pins. Intensities of $\mathrm{Kr}_{2}{ }^{*}$ emissions at $147 \mathrm{~nm}$ were observed through the output coupler with and without the back reflector and represents in Fig. 4 as a function of $\mathrm{Kr}$ gas pressure. Although laser oscillation was

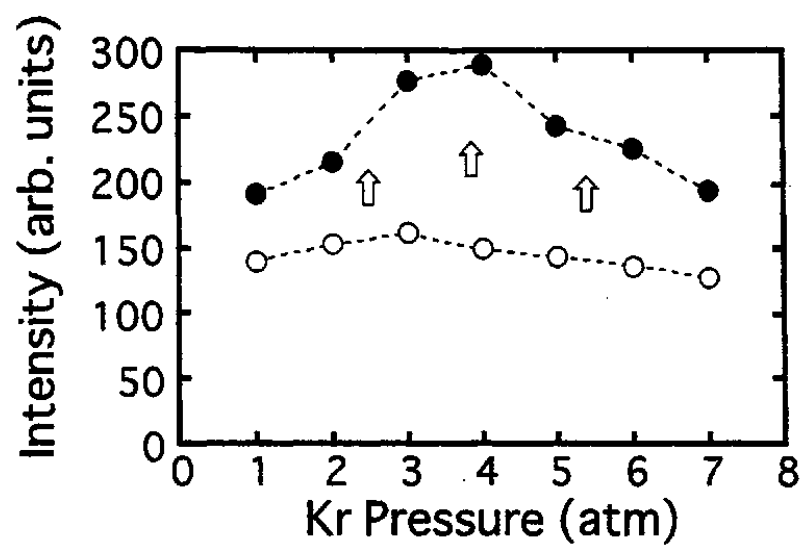

Fig. 4. $147 \mathrm{~nm} \mathrm{Kr}$ emissions intensities.
: With cavity resonator.
$O$ : Without cavity resonator.

not observed, it seemed that laser gains increased with increasing the $\mathrm{Kr}$ pressure up to $4 \mathrm{~atm}$. When the laser cavity was composed, $\mathrm{Kr}_{2}{ }^{*}$ intensities rapidly increased with $\mathrm{Kr}$ pressure. On the contrary only small change was observed for $\mathrm{Kr}_{2}{ }^{*}$ intensities without the laser cavity. We estimated laser gains by comparing $\mathrm{Kr}_{2}{ }^{*}$ intensities with and without laser cavity and found $2.2 \times 10^{-3} \mathrm{~cm}^{-1}$ at 4 atm. of $\mathrm{Kr}$ gas pressure which was about one half of threshold gain of 5.7 $\times 10^{-3} \mathrm{~cm}^{-1}$ for this laser cavity.

We have obtained laser gain relatively close to the threshold gain, it is, however, difficult to increase the gain in this experimental setup. It was found that plasma impedance became very small value of $0.03 \Omega$, therefore only small amount of charged energy was deposited in the plasma within one cycle of discharge current. According to a computer simulation for electron beam excitation, excitation intensity of about 30 $\mathrm{MW} / \mathrm{cm}^{3}$ should be necessary to lase $\mathrm{Kr}$ excimer 
lasers. Considering differences between an electron beam excitation and a discharge excitation, excitation intensity of $20 \mathrm{MW} / \mathrm{cm}^{3}$ may be required to deposit in the plasma. In these experiments maximum charged energy into the capacitors correspond to $20 \mathrm{MW} / \mathrm{cm}^{3}$ therefore almost all charged energy should be deposited in the discharge plasma to provide laser oscillation.

\section{Jet discharge excimer lamps}

Rare gas excimer discharge lamps have been developed in quasi-cw jet discharges. Since the detail of the experimental setup has been described in the previous paper [11], only brief description is presented here. A Laval nozzle of $0.4 \mathrm{~mm}$ throat diameter was located inside a vacuum chamber and a pair of iron electrodes were placed at $6 \mathrm{~mm}$ below the nozzle end and the distance between the electrodes was $5 \mathrm{~mm}$. The electrodes were connected to a $0.53 \mu \mathrm{F}$ capacitor, which was charged up to $2 \mathrm{kV}$. When laboratory-grade rare gas was injected from the nozzle for $5 \mathrm{~ms}$ (FWHM) the discharge was initiated.

It was verified experimentally that the rare gas excimers were formed directly from relevant rare gas clusters in expanding jet discharges.[11] As listed in Table 2 sizable outputs with considerably high efficiencies were obtained by approximated point sources which might be favorable as a light source for optical lithography. The outputs of excimer lamps were improved by about 2.5 time by applying a magnetic field parallel to the gas jet discharge current. [9] In addition to the well known homo-nuclear rare gas excimer continuum, emission from hetero-nuclear
Table 2. Outputs and efficiencies of jet excimer lamps.

\begin{tabular}{|c|c|c|c|}
\hline Species & $\begin{array}{c}\text { Wavelength } \\
(\mathrm{nm})\end{array}$ & $\begin{array}{c}\text { Output Power } \\
(\mathrm{mW})\end{array}$ & $\begin{array}{c}\text { Efficiency } \\
(\%)\end{array}$ \\
\hline $\mathrm{Ar}_{2}{ }^{*}$ & 126 & 4 & 0.004 \\
\hline $\mathrm{ArKr}$ & 134 & 4 & 0.004 \\
\hline $\mathrm{Kr}_{2}{ }^{*}$ & 147 & 130 & 0.9 \\
\hline $\mathrm{Xe}_{2}{ }^{*}$ & 172 & 210 & 1.6 \\
\hline
\end{tabular}

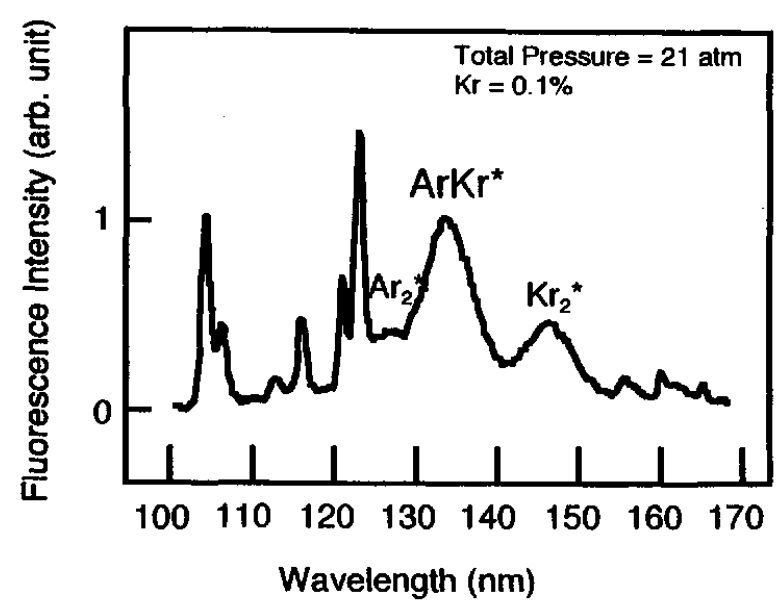

Fig. 5. A hetero-nuclear excimer ArKr* emission spectra from a jet discharge in an argon krypton mixture.

rare gas excimers were obtained by mixing a trace of heavier rare gas. In Fig. 5 a clear ArKr* excimer emission centered at $134 \mathrm{~nm}$ as well as $\mathrm{Ar}_{2}{ }^{*}$ at $126 \mathrm{~nm}$ and $\mathrm{Kr}_{2}{ }^{*}$ at $146 \mathrm{~nm}$ are shown, where $12 \mathrm{mmHg}$ of Krypton gas is mixed with $250 \mathrm{mmHg}$ of argon gas. It should be pointed out that the $134 \mathrm{~nm}$ source may be desirable for future optical lithography, because it is shortest wavelength where refractive optics will be available. 


\section{Summary}

Coherent and incoherent light sources by using rare gas excimers are reviewed from a point of view of post ArF lithography. Laser medium in rare gas excimer lasers are chemically stable which shows a strong contrast to chemically active fluorine in a $F_{2}$ laser, so that rare gas excimer lasers could be a strong candidate for post ArF laser optical lithography. An arc free stable discharge was established in high pressure rare gas up to $10 \mathrm{~atm}$. We have obtained laser gain of $2.2 \times 10^{-3} \mathrm{~cm}^{-1}$ in $\mathrm{Kr}$ discharges which was very close to the threshold gain. An establishment of compact and high repetition operation of a rare gas excimer laser will be essential for semiconductor industry in the next century. On the other hand, a jet excimer lamp would become a competitor to a rare gas excimer laser. Jet excimer lamps work well in discharge excitation with considerably high efficiency and high repetition. Improvements of the emission intensities from jet excimer lamps will be desirable.

\section{References}

1. M.V.McCusker, The Rare Gas Excimers in Excimer Lasers edited by C.K.Rhodes (SpringerVerlag, New York, 1984)

2. Y.Uehara, W.Sasaki, S.Saito, E.Fujiwara,
Y.Kato, M.Yamanaka, K.Tsuchida and J.Fujita, Opt. Lett. 9, (1984) 539.

3. K.Kurosawa, Y.Takikawa, W.Sasaki, M.Okuda, E.Fujiwara, K.Yoshida and Y.Kato, IEEE J. Quamtum Electron. 27, (1991) 71.

4. E.R.Ault et al., ARPA Contract No.0014-72C-0456 (1975)

5. Y.Uehara, W.Sasaki, S.Kasai, S.Saito, E.Fujiwara, Y.Kato, C.Yamanaka, M.Yamanaka, K.Tsuchida and J.Fujita, Opt. Lett. 10, (1985) 487.

6. K.Kurosawa, W.Sasaki, E.Fujiwara and Y.Kato, IEEE J. Quantum Eledtron. 24, (1988) 1908.

7. T.Sakurai, N.Goto and C.E.Webb, J. Phys. D, 20, (1987) 709.

8. K.Mitsuhashi, T.Igarashi, M.Komori, E.Futagami, J.Kawanaka, S.Kubodera, K.Kurosawa and W.Sasaki, Opt. Lett. 20, (1995) 2423.

9. J.Kawanak, A.Ogata, S.Kubodera, W.Sasaki and K.Kurosawa, Appl. Phys., B 65, (1997) 609. 10. S.Kubodera, M.Kitahara, J.Kawanaka, W.Sasaki, and K.Kurosawa, Appl. Phys. Lett., 69, (1996) 452.

11. J.Kawanaka, S.Kubodera, W.Sasaki, K.Kurosawa, K.Mitsuhashi, and T.Igarashi, IEEE J. Select. Topics in Quantum Electron. 1, (1995) 852.

12. C.B.Edwards et al., Rev. Sci. Instrum, 50, (1978) 1201. 